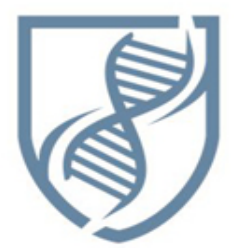

Journal of Bioscience and Applied Research

\section{JBAAR}

WWW.JBAAR.ORG

\title{
Assessment of the hazard effect of an environmental pollutant, (2-OH-BDE 123) using zebrafish embryos
}

\author{
Nessrin A. Kheirallah ${ }^{1 \#}$ and Tamer El-Sayed. Ali $^{2}{ }^{\#}$ \\ ${ }^{1}$ Zoology Department, Faculty of Science, Alexandria University, Egypt. \\ ${ }^{2}$ Oceanography Department, Faculty of Science, Alexandria University, Egypt. \\ \# College of Sciences and Humanity Studies P. O. Box 83, Alkharj 11942, Prince Sattam bin Abdulaziz University, \\ Kingdom of Saudi Arabia. \\ (Corresponding author: E-mail: tameraly@yahoo.com)
}

\begin{abstract}
In the recent decades, many environmental pollutants have received significant attention due to their potential ability to mimic the actions of endogenous estrogens. These pollutants are referred as environmental estrogens and are suspected of causing health effects in both humans and wildlife through disruption of the endocrine system and causing male reproductive dysfunction, thus they are classified as endocrine disrupting compounds (EDCs) such as phenolic metabolites. To monitor general toxicity of this class of EDCs, embryos of zebrafish were exposed to gradual concentrations of 2-OH-BDE 123 (hydroxybrominated diphenyl ethers). Exposures were done by immersion of 1 hour post fertilization (hpf) zebrafish eggs to $72 \mathrm{hpf}$, nominal concentration range of $0.03: 2.5 \mu \mathrm{M}$. Embryos/ larvae were assessed daily for death and structural defects. Results revealed that concentrations from $0.3 \mu \mathrm{M}$ of such metabolites were toxic to the developing zebrafish causing serious morphological alterations and internal deformations. Both toxicity incidence and potency were correlated with the concentration applied. In conclusion, these compounds induced several teratogenic effects. More studies are required for a proper risk assessment and more attention should be given to this class of chemicals in the aquatic environment.
\end{abstract}

Key words: pollutant, toxicity, zebrafish, developmental abnormality, teratogenicity

\section{Introduction}

In recent decades, concern has been increased regarding chemicals in the environment with the capacity to alter normal endocrine function in wildlife. The sources of such chemicals are diverse and effects have been observed in many vertebrates (El-Sayed Ali et al., 2014) Polybrominated diphenyl ethers (PBDEs) are considered a class of such chemicals and are called organohalogens. These organohalogen compounds are produced in large quantities and are used extensively as flame-retardants in a wide range of products being ubiquitous in the environment (van Boxtel et al., 2008). Increasing concentrations of brominated flame retardants and their metabolites in the environment, human food chain and human tissues raise concern about possible neurotoxic effects and endocrine system malfunction (Legler and Brouwer, 2003). In fish, exposure to one of the most prevalent PBDEs in the environment, BDE47, lead to severe abnormalities and may cause mortalities.

Although many PBDEs have not been well studied, new concern has been raised on their hydroxylated congeners. In organisms, PBDEs can be converted mainly to hydroxylated $(-\mathrm{OH})$ forms after biotransformation, and their hydroxylated metabolites are found to be diversed among organisms (Hakk and Letcher, 2003). Hydroxylated polybrominated diphenyl ethers (OH-PBDEs) have been reported to be found in aquatic and terrestrial wild life and human plasma. Many of these compounds are naturally occurring while others are thought to be either metabolic or environmental transformation products of the commercially 
produced PBDEs (Handayani et al., 1997; Malmvärn et al., 2005).

As a great number of PBDE congeners has been detected in a wide variety of wildlife species, including a number of fish species (Marsh et al., 2004; Valters et al., 2005) and also in humans (Inoue et al., 2006). This study focused on the hydroxylated BDE 123 metabolite, an important metabolite within the pool of hydroxylated PBDEs retained in biological systems and have potential as endocrine disrupting chemicals (Hamers et al., 2006; Hamers et al., 2008).

For the purpose of developing a model system to assess responses to chemicals, the zebrafish was selected for the present study due to extensive knowledge of its morphological, biochemical, and physiological characteristics (Kammann et al., 2009; El-Sayed Ali, 2011; El-Sayed Ali and Legler, 2011). The objective of the present study was to determine concentration dependent effects of 2-OH-BDE 123 on zebrafish (Danio rerio) embryos.

\section{Materials and Methods}

\subsection{Chemicals}

Five stock solutions $(0.3,1,3,10$ and $25 \mathrm{mM})$ of 2-OHBDE 123 were dissolved in dimethyl sulfoxide (DMSO, $0.01 \%$ ) immediately prior to use and then directly diluted 10000 times in Dutch standard water (nominal concentrations: $0.03,0.1,0.3,1$ and $2.5 \mu \mathrm{M})$. Solvent (DMSO, 0.01\%) and positive controls (6-OH-BDE 47, its effect on Zebrafish embryos was studied in a preliminary experiment) were incorporated in the experiment.

\subsection{Fish and experimental protocol}

Zebrafish (Danio rerio) were kept under standard laboratory conditions at about $26{ }^{\circ} \mathrm{C} \pm 1{ }^{\circ} \mathrm{C}$ and a photoperiod of 14:10 h. light: dark (Brand et al., 2002) in the Institute of Environmental Studies, Vrije University, Amsterdam, The Netherlands. Fish were fed with dry fish feed, Tetra-Pro Flakes (Tetra GmbH, Germany) in the morning and hatched brine shrimp (Artemia cysts from INVE, Grantsvillle, UT, USA) in the afternoon. The fish were acclimated in glass aquaria containing copper free water. Typically, the eggs were spawned synchronously at dawn of the next morning. One hour later, eggs quality has been checked under the microscope (Leica MZ 75), being sure to select the healthy, fertilized eggs-can be easily identified by their transparency- for the experiment. Fish breeding and embryo manipulation were conducted according to (Westerfield et al., 1997).

\subsection{Toxicity assay}

Selected eggs ( 1 hour post fertilization, hpf) were placed in 24-well cell culture sterilized plates (one embryo/well) with self-adhesive foil. Embryos were exposed to these concentrations at the $4: 8$ - cell stage (1:1.25 hour post fertilization, hpf). Ten embryos/concentration were used and incubated at $26^{\circ} \mathrm{C} \pm 1{ }^{\circ} \mathrm{C}$ for $72 \mathrm{~h}$. Control of the light cycle to $14 \mathrm{~h}$ light and $10 \mathrm{~h}$ dark is achieved by keeping the eggs in a separate room equipped with an automatic light control. Embryos/larvae were screened daily and scored for survival, alterations in morphology, developmental abnormalities and endpoints of toxicity (Nagel, 2002). Toxic/lethal end points (coagulation, missing heartbeat, missing somites, missing tail detachment, missing spontaneous movement) and non-lethal malformations (pericardial or yolk sac oedema, bent notochord, fin malformation, no pigmentation, incomplete head and eye development) were reported separately. The experiment was repeated twice.

Developed embryos/larvae were examined and photographed daily by stereo microscope. Paintshop Pro. 8 image analysis software was utilized to control a Roper digital camera on the microscope. Images were depicted at all treatment levels to complete the picture of the morphological abnormalities in different organs.

\subsection{Calculation of $\mathrm{LC}_{\mathbf{5 0}}$ and $\mathrm{EC}_{\mathbf{5 0}}$}

The $\mathrm{LC}_{50}$ and $\mathrm{EC}_{50}$ were calculated at 96 hours post

fertilization from concentration-\% lethality and concentration-\% effect curves, respectively for all end points separately as well as for the sum of lethal affected embryos.

\section{Results}

The dose-related effects of the metabolite were determined following 72 hour of exposure starting at 1 hour post fertilization (hpf). Respecting to the lethal action and teratogenic potentialof such metabolite, it was highly concentration dependent and partially time dependent. The results showed a very high teratogenic potential for this compound related to the levels(Figures 1-3).

For the groups subjected to 0.03 or $0.1 \mu \mathrm{M}$ 2-OH-BDE 123 , no effects were reported during all the experimental period, presenting embryos similar to those of the DMSO control group during the experimental period. However, $0.3 \mu \mathrm{M}$ subjected group was going as the previously mentioned group till 24 hour, then non-lethal abnormalities in $80 \%$ of embryos were recorded at $48 \mathrm{hpf}$. Respecting to the concentrations 1 and $2.5 \mu \mathrm{M}$, they were lethal within the first three hours of exposure, all embryos stopped their development in the epiboly stage. The developmental effects of 2-OH-BDE 123were dose dependent with an $\mathrm{EC}_{50}$ value of $0.35 \mu \mathrm{M}$ for all endpoints and $\mathrm{LC}_{50}$ of $0.9 \mu \mathrm{M}$. Embryos exposed to 6-OH-BD 47, showing slow developed embryos with neither heart beat nor detached tail from the beginning and being in this form during all the test period without further growth, it's used only for checking the validity of the test condition during the study period.

\section{Effect of exposure to $24 \mathrm{hpf}$}

From morphological points of view, no alterations in the embryos were reported when concentrations of $0.03,0.1$ and $0.3 \mu \mathrm{M}$ were applied, compared with those of the DMSO control group during the first $24 \mathrm{~h}$ of development, showing well developed healthy embryos with somites, yolk sac, detached tail, head, eyes, prominently sculptured brain and few pigment cells are present along the axis dorsal to the yolk extension and on the dorsal part of the yolk ball (Figure 1, A), similar to the control ones (Figure 1, C). While, embryos exposed to 1 
and $2.5 \mu \mathrm{M}$ showed coagulation at the beginning (Figure 1, B). Embryos exposed to 6-OH-BD 47, showed slow developed embryos with neither heart beat nor detached tail (Figure 1, D).

\section{Effect of exposure to $48 \mathrm{hpf}$}

Embryos exposed to concentrations of 0.03 and $0.1 \mu \mathrm{Mof}$ 2-OH-BDE 123, showing embryos with well-developed notochord, otolith, caudal fin, head, eyes and pigment extends the whole length of the body (Figure 2, A), similar to the control group embryos. The $0.3 \mu \mathrm{M}$ treatedgroup showingmal-formed embryos with oedema and a slightly unstraight notochord with mal-formed tail (curved, short, no tail fin) in $80 \%$ of the embryos (Figure $2, \mathrm{~B})$ and $10 \%$ of embryos started to coagulate in the tail region (Figure 2, B'). However, blood circulates through a closed set of channels and clear heart beats were measured and ranged between 119-120 beats/ min., as those who were healthy and presented about $10 \%$ of the embryos (Figure 2, B*). Embryos exposed to 6-OH-BD 47, showing no more growth than those recorded at $24 \mathrm{hpf}$.

Effect of exposure to $72 \mathrm{hpf}$

$50 \%$ of the DMSO exposed group and all the embryos exposed to $0.03 \mu \mathrm{M}$ of 2-OH-BDE 123were hatched and presented larvae with quite elongated pectoral fin buds and vigorous heart beats. Also, it was shown that the yolk sac started to be shrunk making the pericardial cavity more conspicuous, like the (Figure 3, A). The embryos exposed to $0.1 \mu \mathrm{M}$ of 2-OH-BDE 123presented delayed hatched larvae, but the larvae were in a good state but still in the sac, similar to the other $50 \%$ of the control group (Figure 3, B). 70\% of embryos treated with $0.3 \mu \mathrm{M}$ were alive showing malformed hatched larvae with no caudal fin and curved notochord with non-lethal endpoints appeared (odema)(Figure 3, C, C', C"), the rest 30\% were unhatched and started to coagulate in the lower part region associated with severe oedema (Figure 3, C*). Embryos exposed to 6-OH-BD 47, showing no more growth than recorded at $24 \mathrm{hpf}$.

\section{Discussion}

The results of the present study revealed a clear and significant effect of a certain class of polybrominated diphenyl ethers (PBDEs or BDEs) which are widely used in our life as additives in a variety of household and industrial products as flame retardants. Concern over the potential effects of PBDEs on environmental and human health led to a ban on production and usage of the penta-BDE and octa-BDE formulations in Europe in 2004 and a voluntary ban on the production of these formulations in certain American states followed shortly thereafter (Betts, 2008). Acute toxicity tests of $\mathrm{OH}-\mathrm{BDEs}$ with aquatic animals can only be a first step for the assessment of the environmental risk of these chemicals. 6-OH-BDE47 was highly toxic to zebrafish Danio rerio with multiple manifestations of toxicity, such as lethal to the adult and embryonic fish and inducing several teratogenic effects such as malformations, scoliosis and oedema to the embryos (van Boxtel et al., 2008).
Based on the findings of 6-OH-BDE47 (van Boxtel et al., 2008), it is plausible that other OH-BDEs may also have a common mode of action, due to the similarities in their molecular structures. Furthermore, little information is known about the potential of such metabolites to accumulate in organisms, and it has been suggested that continuous exposure may result in tissue retention of phase I (hydroxylated) metabolites (Betts, 2008; El-Sayed Ali at al., 2013; El-Sayed Ali and Kheirallah, 2015).

In a study of the effects of hydroxylated organohalogens on endocrine disrupting effects, Certain study by Kester (2000) proposed that the cooperative effect of $-\mathrm{OH}$ and $-\mathrm{Br}$ groups could lead to different potencies of endocrine disruptors. This may give some hints that the position of hydroxyl group is not the only determinant for the uncoupling activity of OH-BDEs, the relative position of substituted bromine atom is also an important factor. Future chemical studies on OH-BDEs may help to understand these structure-specific activities.

The present study confirms that 2-OH-BDE 123can cause lethal sudden effect at the beginning of exposure to doses equal or more than $1 \mu \mathrm{M}$. Meanwhile, developmental abnormalities in zebrafish embryos were shown at doses lower than $1 \mu \mathrm{M}$. Such lower doses were far less toxic than their equivalents of 6-OH-BDE47 which had an action in earlier stages than shown in the present study. The abnormalities were related to the concentration and exposure period to such compound. Growth retardation, malformations of yolk, heart, tail and head were also recorded $^{1}$ by applying 6-OH-BDE47 on zebrafish embryos but still at doses lower than that used in the present study. Under the pressure of oedema, heart can be malformed during development. The increasing pressure can also reduce heartbeat and blood circulation, which finally leads to death (Canton et al., 2005; Canton et al., 2008).

The present study reveals, for the first time, the teratogenic effect of 2-OH-BDE 123and focusing on the toxicity of such metabolites in living organism. Generally, there is a scarce of information about the toxic effect of phenolic PBDE in living organism (El-Sayed Ali, 2011). Further studies are required to follow up the mode of action of such compound although the findings of the present study put a possibility of similarities of action between 2-OH-BDE 123and 6-OH-BDE 47.

Moreover, this study raises the issue of the environmental risks of OHBDEs. In addition, more research into the environmental levels and toxicity of phenolic PBDEs is required to confirm the risks of different classes of $\mathrm{OH}$ BDEs on various organisms.

\section{References}

Betts, K. S. (2008). Unwelecome guest: PBDES in indoor dust. Environmental Health Perspectives, 116: A202-A208.

Brand, M., Granato, M., Nüsslein-Volhard, C. (2002). Zebrafish: A Practical Approach: Keeping and raising zebrafish, IRL Press, Oxford, pp. 261. 

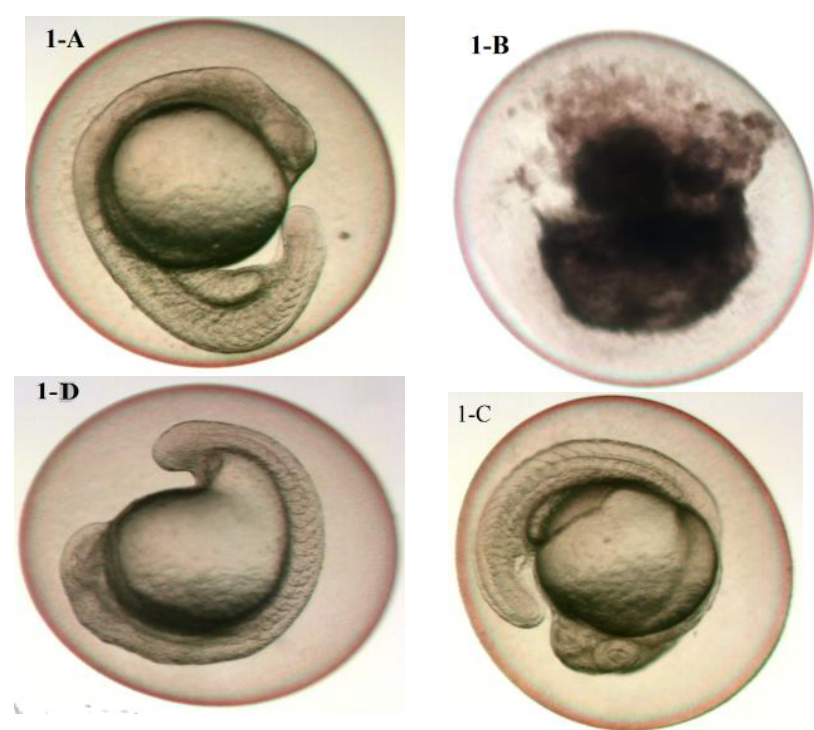

Fig. 1. Changes in the morphology zebrafish embryos exposed to different concentrations of 2-OH-BDE 123 and were photographed live in lateral orientation through a stereomicroscope at $24 \mathrm{~h}$ post fertilization (hpf). Embryos exposed to concentrations of $0.03,0.1$ and $0.3 \mu \mathrm{M}$, showing well developed embryo with yolk sac, tail, head, eyes and pigmentation similar to the control group embryos (1-A) and identical to those subjected to DMSO (1-C). Embryos exposed to 1 and $2.5 \mu \mathrm{M}$ showing death at the beginning (coagulate, 1-B). Embryos exposed to 6OH-BDE 47, showed slow developed embryos with neither heart beat nor detached tail (1-D) (×4).
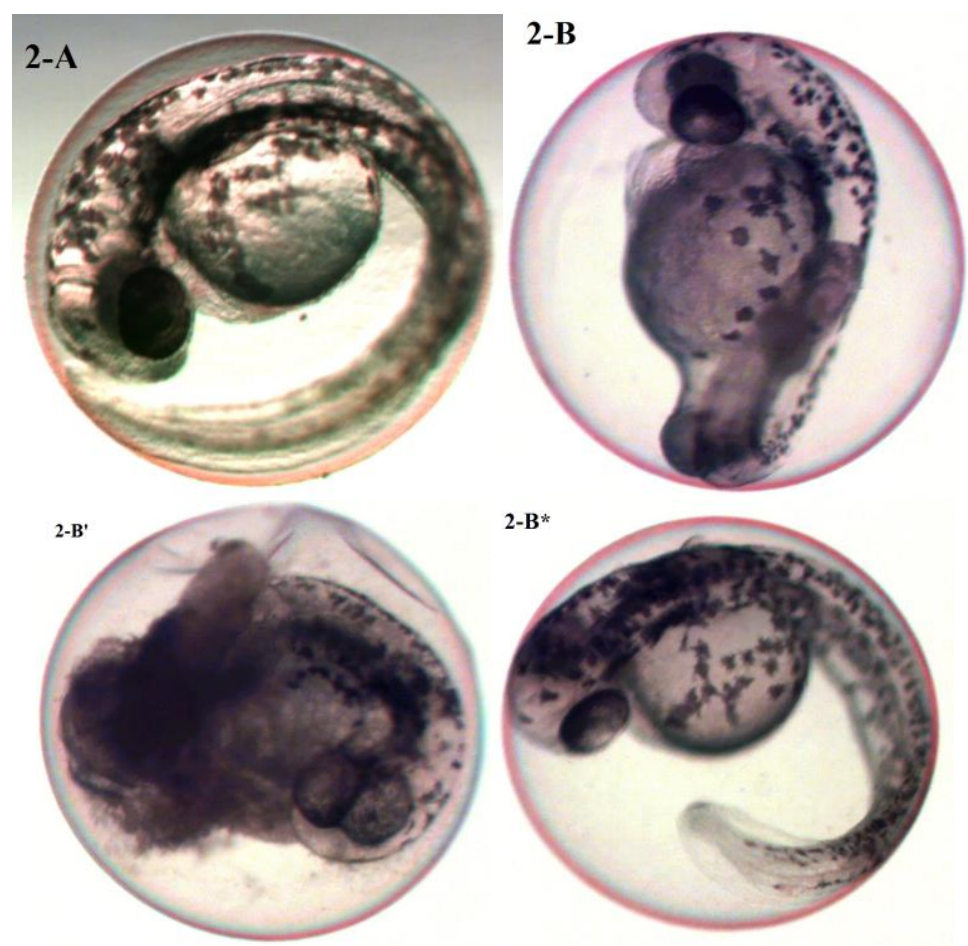

Fig. 2. Morphological alterations in zebrafish embryos exposed to different concentrations of 2-OH-BDE 123 and were photographed live through a stereomicroscope at $48 \mathrm{~h}$ post fertilization (hpf). Embryos exposed to concentrations of 0.03 and $0.1 \mu \mathrm{M}$, showing embryos with well developed notochord with muscles, otolith, caudal fin, head, eyes and pigmentation similar to the control group embryos (2-A). $0.3 \mu \mathrm{M}$ treated group, $80 \%$ of them showing mal-formed embryos with mal-formed short tail with no tail fin, severe oedema is also observed (2-B), $10 \%$ of the embryos started to coagulate in the lower part region (2-B'), and other $10 \%$ of the embryos are healthy(2-B*), $(\times 4)$. 

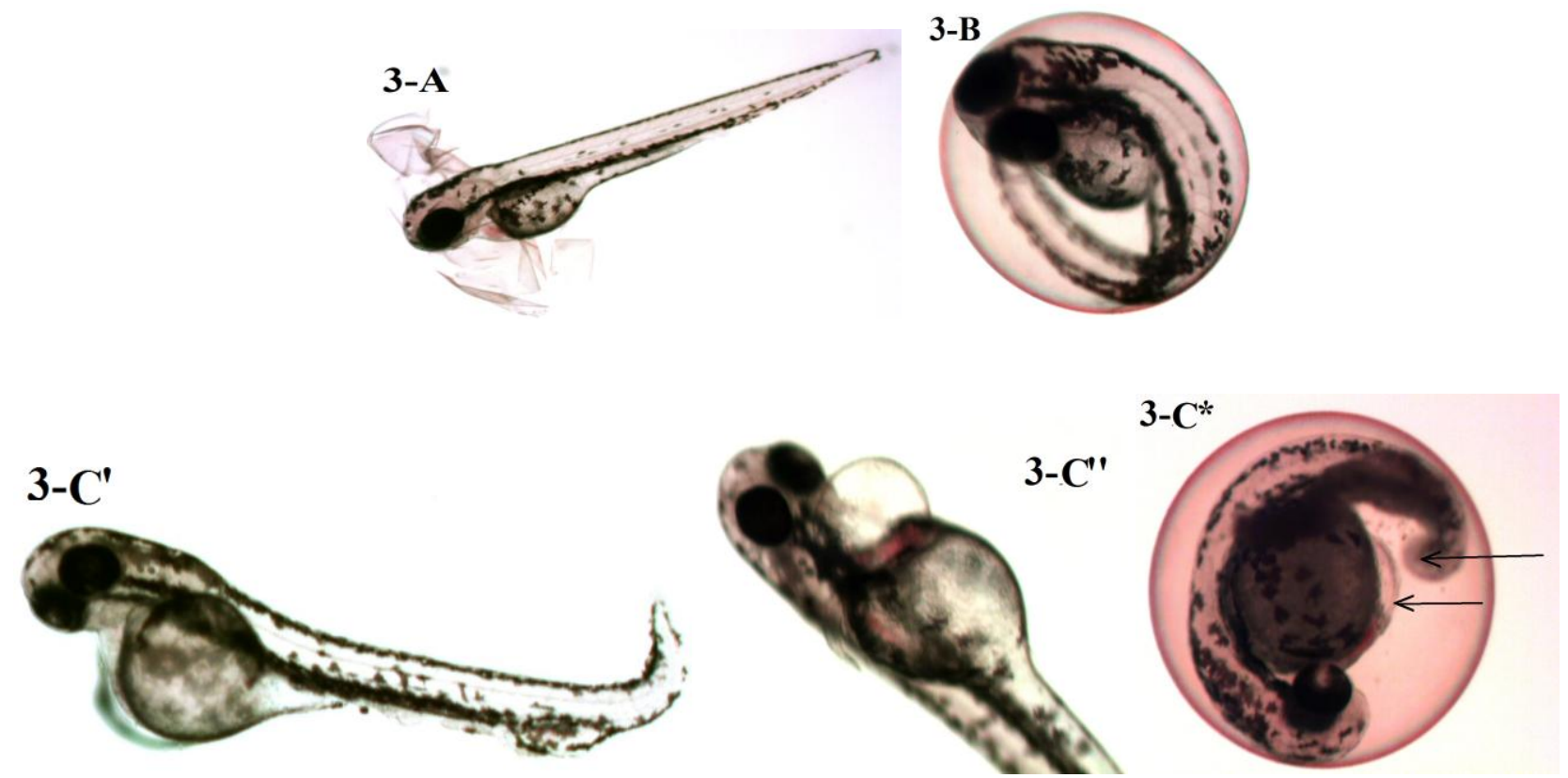

Fig. 3. Morphological changes in zebrafish embryos exposed to different concentrations of 2-OH-BDE 123 and were photographed live in lateral orientation through a stereomicroscope at $72 \mathrm{~h}$ post fertilization (hpf). Embryos exposed to concentrations of $0.1 \mu \mathrm{M}$ showing well developed hatched larvae similar to the control group larvae (only $50 \%$ of the control group were hatched) $(3-\mathrm{A}, \times 2)$, hatching was shown in all embryos of these groups. Embryos exposed to concentrations of $0.03 \mu \mathrm{M}$ showing well developed unhatched larvae similar to the control group larvae (3-B, $\times 4) 0.03$. $50 \%$ of the control- DMSO- treated group was healthy hatched larvae while the rest was healthy unhatched larvae. 0.3 $\mu \mathrm{M}$ treated group, $70 \%$ of them were hatched, showing mal-formed larvae with curved tail end with no tail fin (3-C', $\times 2)$, severe oedema is also observed (3-C", $\times 2$ ), 30\% of the embryos were unhatched started to coagulate in the lower part region associated with severe oedema (arrows, 3-C*, $\times 4$ ).

Canton, R. F., Sanderson, J. T., Letcher, R. J., Bergman, A., van den Berg, M. (2005). Inhibition and induction of aromatase (CYP19) activity by brominated flame retardants in H295R human adrenocortical carcinoma cells. Toxicological Sciences, 88: 447-455.

Canton, R. F., Scholten, D. E., Marsh, G., de Jong, P. C., van den Berg, M. (2008). Inhibition of human placental aromatase activity by hydroxylated polybrominated diphenyl ethers (OH-PBDEs). Toxicology and Applied Pharmacology, 227: 68-75.

El-Sayed Ali, T. (2011). The cytotoxic effects of 5methoxylated polybrominated diphenyl ether 47 (BDE 47) on zebrafish (Danio rerio) embryos. Egyptian Journal of Aquatic Research, 37: 87-93.

El-Sayed Ali, T. (2011). The cytotoxic effects of 5methoxylated polybrominated diphenyl ether 47 (BDE 47) on zebrafish (Danio rerio) embryos. Egyptian Journal of Aquatic Research, 37: 87-93.

El-Sayed Ali, T., Legler, J. (2011). Developmental toxicity of nonylphenol in zebrafish (Danio rerio) embryos. Indian Journal of Marine Sciences, 40: 509-515.

El-Sayed Ali, T., Kheirallah, N. (2015). Toxic effects of phenolic metabolite 6-OH-BDE 85 on zebrafish (Danio rerio) embryos. Indian Journal of Geo-Marine Sciences, In press.
El-Sayed Ali, T., Al-Ghanim, K., Legler, J. (2013). Novel non-estrogenic endpoints of alkylphenol toxicity in fish. Indian Journal of Marine Sciences, 42: 770-774.

El-Sayed Ali, T., Abdel-Aziz, S. H., El-Sayed, A. M., Zeid, S. (2014). Structural and functional effects of early exposure to 4-nonylphenol on gonadal development of Nile tilapia (Oreochromis niloticus): a-histological alterations in testes. Fish Physiology and Biochemistry, 40: 1495-1507.

Hakk, H., Letcher, R. J. (2003). Metabolism in the toxicokinetics and fate of brominated flame retardants-a review. Environment International, 29: 801-828.

Hamers, T., Kamstra, J. H., Sonneveld, E., Murk, A. J., Kester, M. H., Andersson, P. L., Legler, J., Brouwer, A. (2006). In vitro profiling of the endocrinedisrupting potency of brominated flame retardants. Toxicological Sciences, 92: 157-173.

Hamers, T., Kamstra, J. H., Sonneveld, E., Murk, A. J., Visser, T. J., van Velzen, M. J. M., Brouwer, A., Bergman, A. (2008). Biotransformation of brominated flame retardants into potentially endocrine-disrupting metabolites, with special attention to 2, 2', 4, 4'tetrabromodiphenyl ether (BDE-47). Molecular Nutrition \& Food. Research, 52: 284-298.

Handayani, D., Edrada, R. A., Proksch, P., Wray, V., Witte, L., van Soest, R. W., Kunzmann, A. (1997). 
Soedarsono Four new bioactive polybrominated diphenyl ethers of the sponge Dysidea herbacea from West Sumatra, Indonesia. Journal of Natural Products, 60: 1313- 1316.

Inoue, K., Harada, K., Takenaka, K., Uehara, S., Kono, M., Shimizu, T., Takasuga, T., Senthilkumar, K., Yamashita, F., Koizumi, A. (2006). Levels and concentration ratios of polychlorinated biphenyls and polybrominated diphenyl ethers in serum and breast milk in Japanese mothers. Environmental Health Perspectives, 114: 1179-1185.

Kammann, U., Vobach, M., Wosniok, W., Schäffer, A., Telscher, A. (2009). Acute toxicity of 353nonylphenol and its metabolites for zebrafish embryos. Environmental Science \& Pollution Research, 16: 227231.

Kester, M. H., Bulduk S, Tibboel, D., Meinl, W., Glatt, H., Falany, C. N., Coughtrie, M. W., Bergman, A., Safe, S. H., Kuiper, G. G., Schuur, A. G., Brouwer, A., Visser, T. J. (2000). Potent inhibition of estrogen sulfotransferase by hydroxylated PCB metabolites: a novel pathway explaining the estrogenic activity of PCBs. Endocrinology, 141: 1897-1900.

Legler, J., Brouwer, A. (2003). Are brominated flame retardants endocrine disruptors?'. Environment International, 29: 879-885.

Malmvärn, A., Marsh, G., Kautsky, L., Athanasiadou, M., Bergman, A., Asplund, L. (2005). Hydroxylated and methoxylated brominated diphenyl ethers in the red algae Ceramium tenuicorneand and blue mussels from the Baltic Sea. Environmental Science \& Technology, 39: 2990-2997.

Marsh, G., Athanasiadou, M., Bergman, A., Asplund, L. (2004). Identification of hydroxylated and methoxylated polybrominated diphenyl ethers in Baltic Sea salmon (Salmo salar) blood. Environmental Science \& Technology, 38: 10-18.

Nagel, R (2002). DarT: the embryo test with the zebrafish Danio rerio - a general model in ecotoxicology and toxicology. Altex-Altern Tierexp, 19: 38-48.

Westerfield, M., Doerry, E., Kirkpatrick, A. E., Driever, W., Douglas, S. A. (1997). An on-line database for zebrafish development and genetics research. Seminars in Cell Developmental Biology, 8: 477-4.

Valters, K., Li, H., Alaee, M., D’Sa, I., Marsh, G., Bergman, A., Letcher, R. J. (2005). Polybrominated diphenyl ethers and hydroxylated and methoxylated brominated and chlorinated analogues in the plasma of fish from the Detroit River. Environmental Science \& Technology, 39: 5612-5619.

van Boxtel, A., Kamstra, J. H., Cenijn, P. H., Pieterse, B., Wagner, M. J., Antink, M., Krab, K., van der Burg, B., Marsh, G., Brouwer, A., Legler, J. (2008). Microarray analysis reveals a mechanism of phenolic polybrominated diphenylether toxicity in Zebrafish. Environmental Science \& Technology, 42: 1773-1779. 